\title{
CORRECÇÃO DE ESCOLIOSE LOMBAR DEGENERATIVA POR TÉCNICA MINIMAMENTE INVASIVA
}

\author{
CORRECTION OF DEGENERATIVE LUMBAR SCOLIOSIS BY MINIMALLY \\ INVASIVE TECHNIQUE
}

\author{
CORRECCIÓN DE ESCOLIOSIS LUMBAR DEGENERATIVA POR TÉCNICA \\ MÍNIMAMENTE INVASIVA
}

Pedro Santos Silva', Joana Silva ${ }^{1}$, Bruno Carvalho ${ }^{1}$, Paulo Pereira ${ }^{2}$, Rui Vaz ${ }^{3}$

\section{RESUMO}

A terapêutica cirúrgica da escoliose degenerativa consiste habitualmente em instrumentações e fusões mais ou menos extensas e associa-se a morbilidade significativa. A evolução tecnológica tem aberto caminho a técnicas menos invasivas que permitem obter resultados sobreponíveis aos das técnicas tradicionais minimizando a agressão cirúrgica. Descreve-se o caso de uma paciente do sexo feminino, de 63 anos, submetida a descompressão lombar posterior, em Janeiro de 2009, por alterações degenerativas marcadas. Poucos meses após a cirurgia a paciente referiu aumento das lombalgias e ciatalgia direita. $\mathrm{O}$ estudo imagiológico demonstrou agravamento de escoliose degenerativa L2-L5 associada a extrusão discal L2-L3 direita calcificada, fractura bilateral dos pedículos de L3 e espondilolistese degenerativa grau 1 L5-S1. Foi submetida a reintervenção cirúrgica por técnica minimamente invasiva consistindo em TLIF's L2-L3, L3-L4, L4-L5 e L5-S1 e fixação transpedicular L2-S1 bilateral, com correcção da deformidade no plano sagital e coronal. O caso clínico apresentado ilustra o potencial das abordagens minimamente invasivas no tratamento cirúrgico de escolioses degenerativas, devendo ser uma opção sempre presente considerando os benefícios potenciais para o paciente.

Descritores: Escoliose/cirurgia; Procedimentos cirúrgicos minimamente invasivos; Relatos de casos.

\begin{abstract}
Surgical treatment of degenerative scoliosis usually consists of more or less extensive instrumentation and fusion and is associated with significant morbidity. Technological developments have opened the way for less invasive techniques which allow obtaining results comparable to traditional techniques, minimizing surgical trauma. In this work, we describe the case of a 63-year-old female patient who underwent posterior lumbar decompression, in January 2009, due to marked degenerative changes. A few months after surgery the patient reported increased back pain and right sciatica. Imaging studies showed exacerbation of L2-L5 degenerative scoliosis associated with L2-L3 right calcified disc extrusion, bilateral fracture of the pedicles of L3 and L5-S1 grade 1 degenerative spondylolisthesis. She underwent another surgery by minimally invasive technique with TLIF of L2-L3, L3-L4, L4-L5 and L5-S1 and bilateral L2-S1 transpedicular fixation with correction of deformity in sagittal and coronal planes. This case report illustrates the potential of minimally invasive approaches in the surgical treatment of degenerative scoliosis and should always be an option considering the benefits to the patient.
\end{abstract}

Keywords: Scoliosis/surgery; Surgical procedures, minimally invasive; Case reports.

\section{RESUMEN}

El tratamiento quirúrgico de la escoliosis degenerativa consiste habitualmente en instrumentaciones y fusiones más o menos extensas, asociadas a morbilidad significativa. La evolución tecnológica ha abierto el camino para técnicas menos invasivas que permiten obtener resultados comparables a los de las técnicas tradicionales, minimizando el trauma de la cirugía. Presentamos el caso de paciente de sexo femenino, de 63 años de edad, sometida a descompresión lumbar posterior en enero de 2009, debido a marcadas alteraciones degenerativas. Pocos meses después del procedimiento, la paciente refirió agravamiento de la lumbalgia y ciática derecha. El estudio de imágenes mostró empeoramiento de la escoliosis degenerativa L2-L5 asociada a extrusión discal L2-L3 derecha calcificada, fractura bilateral de los pedículos de L3 y espondilolistesis degenerativa grado I L5-S1. Fue sometida a la reintervención quirúrgica utilizando una técnica mínimamente invasiva que consistió en TLIF L2-L3, L3-L4, L4-L5 y L5-S1 y fijación transpedicular L2-S1 bilateral, con corrección de la deformidad en los planos sagital y coronal. El caso clínico que presentamos ilustra el potencial de los enfoques mínimamente invasivos en el tratamiento quirúrgico de las escoliosis degenerativas, debiendo ser una opción siempre presente cuando se consideran los beneficios para el paciente.

Descriptores: Escoliosis/cirugía; Procedimientos quirúrgicos mínimamente invasivos; Informes de casos.

1. Interno de Formação Específica de Neurocirurgia do Serviço de Neurocirurgia do Hospital de São João - Porto, Portugal.

2. Assistente Hospitalar de Neurocirurgia do Hospital de São João; Assistente Convidado de Neurologia e Neurocirurgia da Faculdade de Medicina da Universidade do Porto - FMUP - Portugal.

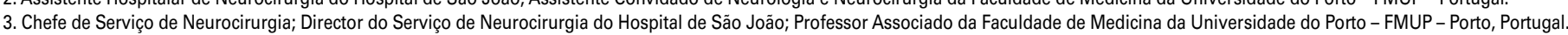




\section{INTRODUÇÃO}

A escoliose degenerativa é uma patologia comum da coluna vertebral associada à morbilidade significativa, ocorrendo em $6 \%$ da população com mais de 50 anos $^{1}$. Caracteriza-se por uma deformidade com um ângulo de Cobb superior a $10^{\circ}$ no plano coronal, atingindo mais frequentemente a coluna lombar². Tem origem em fenómenos degenerativos articulares assimétricos que condicionam desvios frontais ou sagitais da coluna que por sua vez aumentam a instabilidade vertebral e favorecem a progressão da doença, fechando assim um círculo vicioso ${ }^{2}$. A apresentação clínica típica consiste em lombalgia, frequentemente associada a dor radicular e claudicação neurogénica ${ }^{3}$

A maioria dos casos de escoliose degenerativa lombar é abordada de forma conservadora ${ }^{4}$. A terapêutica cirúrgica é reservada para os casos de dor refractária a tratamento médico, défice neurológico maior ou progressivo, agravamento documentado da deformidade e instabilidade vertebral5 ${ }^{5,6}$. As técnicas mais utilizadas consistem em fusão vertebral apoiada em fixação transpedicular.

Neste trabalho, descreveu-se o caso de uma paciente submetida à correcção de escoliose lombar degenerativa por técnica minimamente invasiva

\section{CASO CLÍNICO}

Paciente do sexo feminino, de 63 anos, com antecedentes de artrite reumatóide (em corticoterapia e metotrexato), HTA e nefrectomia direita.

Em Janeiro de 2009 foi submetida a descompressão lombar posterior com hemilaminectomia direita de L3, remoção de sequestro discal calcificado paramediano direito, flavectomia L4-L5 esquerda com fenestração parcial da lâmina de L4 e remoção de hérnia calcificada L4-L5.

Após evolução pós-operatória favorável referiu, em Setembro de 2009, agravamento progressivo do quadro prévio, com dor de predomínio no membro inferior direito, resistente a analgésicos opióides. As dificuldades na marcha acentuaram-se gradualmente, com necessidade inicial de recorrer a apoio externo e com impossibilidade de deambulação a partir de Dezembro. Apresentava pontuação de 9/10 na Escala Visual Analógica de Dor (EVA) e 88\% no Índice de Incapacidade de Oswestry (ODI - Oswestry disability index).

No exame neurológico mantinha-se sem défice motor ou sensitivo objectivável, sem alterações nos reflexos osteotendinosos e com sinal de Lasègue negativo bilateralmente.

O estudo imagiológico demonstrou agravamento de escoliose

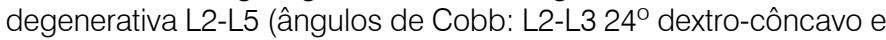
L3-L5 $20^{\circ}$ dextro-convexo) associada a extrusão discal L2-L3 direita parcialmente calcificada, fractura bilateral dos pedículos de L3 e espondilolistese degenerativa grau 1 de L5-S1 (Figura 1).

Foi submetida, em Janeiro de 2010, a reintervenção cirúrgica por técnica minimamente invasiva (Figura 2) consistindo em TLIF
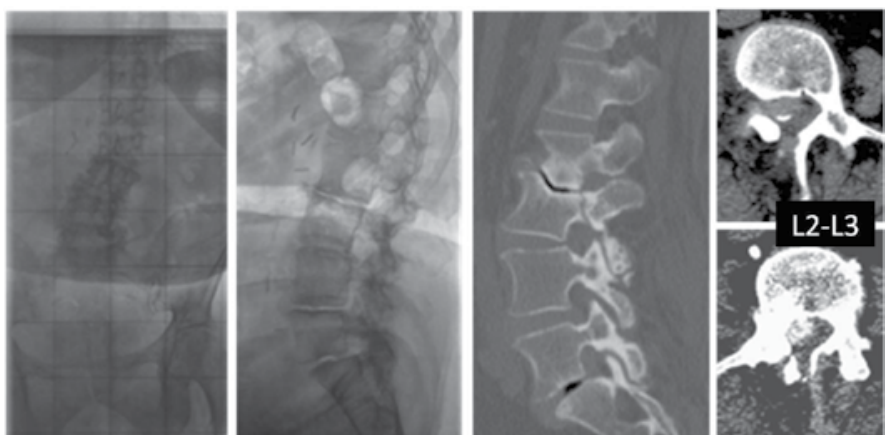

Figura 1 - Radiografia e TC lombares pré-operatórios (Janeiro de 2010).
L2-L3 direito com remoção de material extruso calcificado, TLIF's L3-L4, L4-L5 e L5-S1 esquerdos e fixação transpedicular percutânea L2-S1 bilateral, com correcção da deformidade no plano sagital e coronal. Foi utilizado um retractor expansível Pipeline ${ }^{\text {TM }}$ (DePuy Spine, Johnson \& Johnson) nos acessos minimamente invasivos para realização dos TLIF's, "cages" intersomáticas Concorde ${ }^{\mathrm{TM}}$ Bullet (DePuy Spine, Johnson \& Johnson) preenchidas com osso autólogo local e instrumentação transpedicular percutânea com VIPER ${ }^{\text {TM } 2}$ System (DePuy Spine, Johnson \& Johnson) (Figura 3).

A cirurgia demorou oito horas. Não houve necessidade de transfusão de sangue no perioperatório.

A evolução clínica pós-operatória foi favorável com maior controlo das queixas álgicas lombares e no membro inferior direito (2/10 na VAS). A paciente apresenta actualmente um ODI de 50\%.

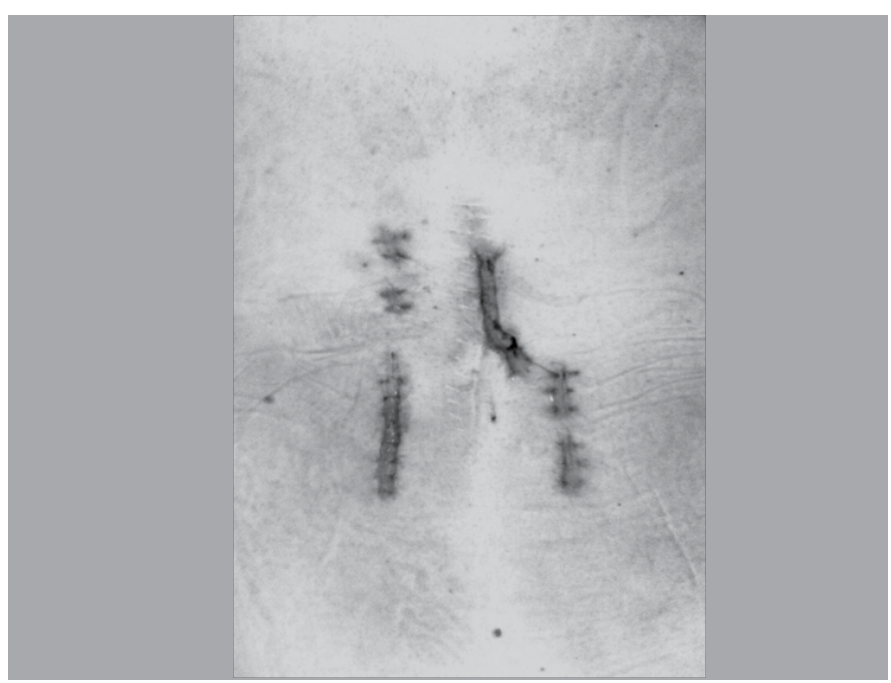

Figura 2 - Incisões lombares ilustrando o acesso.minimamente invasivo.
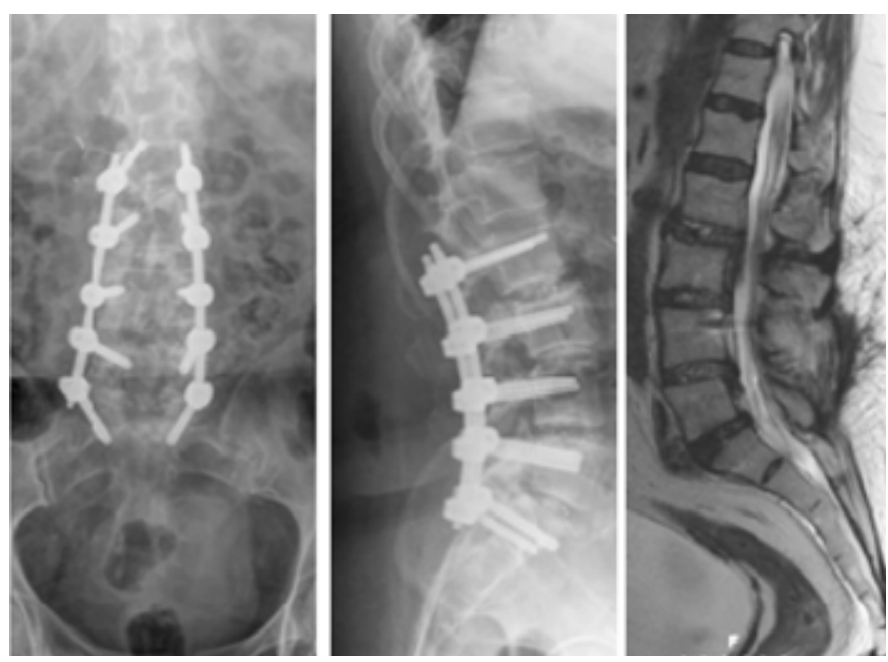

Figura 3 - Estudo imagiológico pós-operatório.

\section{DISCUSSÃO}

A escoliose lombar degenerativa afecta com frequência pacientes idosos com múltiplas comorbilidades e exige, em regra, procedimentos extensos com perdas hemáticas importantes. Em consequência, a terapêutica cirúrgica associa-se a taxas de complicações consideráveis (de 28 a 86\%) ${ }^{7}$.

Nesse contexto, as técnicas minimamente invasivas contribuem para minimizar a morbilidade cirúrgica perioperatória. Além disso, 
facilitam a mobilização precoce e diminuem a dor pós-operatória com limitação do consumo de analgésicos, permitindo reduzir o tempo de internamento ${ }^{7}$. Na única série que descreve a utilização de técnicas minimamente invasivas no tratamento da escoliose lombar degenerativa em vários níveis, Anand et al. ${ }^{8,9}$ documentam o benefício da abordagem minimamente invasiva por meio de uma menor lesão tecidual e da redução das perdas hemorrágicas, sem prejuízo da eficácia terapêutica comparativamente às técnicas abertas tradicionais. Por sua vez, Ringel et al. ${ }^{10}$ relatam a segurança da fixação transpedicular por técnica percutânea na coluna lombar e torácica, mantendo um elevado nível de precisão no posicionamento dos parafusos pediculares.

\section{CONCLUSÕES}

A escoliose degenerativa é uma patologia complexa que permanece um desafio na cirurgia da coluna vertebral.

Uma intervenção cirúrgica neste contexto tem como objectivo o alívio da dor e a correcção da deformidade, com uma fusão sólida.

As técnicas minimamente invasivas são uma alternativa válida e com importância crescente; minimizam o trauma cirúrgico e diminuem a morbilidade.

O caso clínico apresentado ilustrou o potencial das abordagens minimamente invasivas no tratamento cirúrgico de escolioses degenerativas, devendo ser uma opção sempre presente considerando os benefícios potenciais para o paciente.

\section{REFERÊNCIAS}

1. Vanderpool DW, James JI, Wynne-Davies R. Scoliosis in the elderly. J Bone Joint Surg Am. 1969;51(3):446-55.

2. Aebi M. The adult scoliosis. Eur Spine J. 2005;14(10):925-48.

3. Winter RB, Lonstein JE, Denis F. Pain patterns in adult scoliosis. Orthop Clin North Am. 1988; 19:339-45.

4. Tribus CB. Degenerative lumbar scoliosis: evaluation and management. J Am Acad Orthop Surg. 2003;11(3):174-83.

5. Herkowitz HN, Sidhu KS. Lumbar spine fusion in the treatment of degenerative conditions: current indications and recommendations. J Am Acad Orthop Surg. 1995;3(3):123-35.

6. Birknes JK, White AP, Albert TJ, Shaffrey $\mathrm{Cl}$, Harrop JS. Adult degenerative scoliosis: a review. Neurosurgery. 2008;63(3 Suppl):94-103.
7. Wang MY, Anderson DG, Poelstra KA, Ludwig SC. Minimally invasive posterior fixation. Neurosurgery. 2008;63(3 Suppl):197-203.

8. Anand N, Baron EM, Thaiyananthan G, Khalsa K, Goldstein TB. Minimally invasive multilevel percutaneous correction and fusion for adult lumbar degenerative scoliosis: a technique and feasibility study. J Spinal Disord Tech. 2008;21(7);459-67.

9. Anand N, Rosemann R, Khalsa B, Baron EM. Mid-term to long-term clinical and functional outcomes of minimally invasive correction and fusion for adults with scoliosis. Neurosurg Focus. 2010;28(3):E6.

10. Ringel F, Stoffel M, Stüer C, Meyer B. Minimally invasive transmuscular pedicle screw fixation of the thoracic and lumbar spine. Neurosurgery. 2006:59 (4 Suppl 2):ONS361-6. 